\title{
Prevalence and distribution of human papillomavirus genotypes among women in northern Henan Province of China
}

\author{
Xiangpeng Wang \\ Xinxiang Medical University https://orcid.org/0000-0003-2841-876X \\ Yuan Song \\ Xinxiang Medical University \\ Xiaofei Wei \\ Xinxiang Medical University \\ Guanyu Wang \\ Xinxiang Medical University \\ Ruili Sun \\ Xinxiang Medical University \\ Mingyong Wang \\ Xinxiang Medical University \\ Lijun Zhao ( $\sim$ zhaolj2019@126.com ) \\ Xinxiang Medical University https://orcid.org/0000-0002-0906-8314
}

\section{Research}

Keywords: human papillomavirus, genotype, cervical cancer, vaccine

Posted Date: September 15th, 2021

DOI: https://doi.org/10.21203/rs.3.rs-889505/v1

License: (c) (i) This work is licensed under a Creative Commons Attribution 4.0 International License. Read Full License 


\section{Abstract \\ Background}

Human papillomavirus (HPV) infection can cause cervical and other cancers including cancer of vulva, vagina, penis, anus, or oropharynx. However, data concerning the prevalence and genotype distribution of HPV among women are limited in northern Henan Province of China. This study aimed to make an investigation on the current prevalence and genotype distribution of HPV among women and provided comprehensive data to guide HPV-based cervical cancer prevention in northern Henan Province.

\section{Methods}

A total of 15616 women aged 16 to 81 years who attended the department of gynecology of Xinxiang central hospital between January 2018 and December 2019 were enrolled in this study. HPV DNA was detected by PCR method followed by HPV type-specific hybridization. The overall prevalence, age-specific prevalence and genotype distribution of HPV were investigated.

\section{Results}

The overall HPV prevalence was 19.7\% among women in northern Henan Province. Single, double and multiple HPV infections accounted for $13.7 \%, 4.3 \%$ and $1.8 \%$ of the total cases, respectively. HPV prevalence was $41.8 \%$ among women aged 16 to 19 years, $21.1 \%$ among women aged 20 to 29 years, $18.1 \%$ among women aged 30 to 39 years, $17.7 \%$ among women aged 40 to 49 years, $20.8 \%$ among women aged 50 to 59 years, $22.9 \%$ among women more than 60 years. HPV infection rates differed significantly across different age groups. Most infections were caused by high-risk HPV (HR-HPV) and single genotype HPV infection was the most common pattern. The most common HRHPV genotype was HPV16, followed by HPV52, HPV58, HPV53 and HPV39. The most common low-risk HPV (LR-HPV) genotype was HPV6, followed by HPV61, HPV81, HPV54 and HPV11.

\section{Conclusions}

HPV infection is common among women in northern Henan Province. The highest infection prevalence was found in women less than 20 years old. The 9-valent HPV vaccine for routine vaccination is strongly recommended in northern Henan Province.

\section{Introduction}

Cervical cancer, ranked after breast cancer, colorectal cancer and lung cancer, is the fourth most common cancer among women worldwide, with approximately 530,000 new cases and 275,000 deaths every year [1, 2, 3]. According to current data, nearly $85 \%$ of the women deaths from cervical cancer occurred in developing or underdeveloped countries[1]. In China, cervical cancer ranked as the eighth most common killer for women and continued to be a public health problem affecting women health[4]. Persistent infection with HR-HPV is the major cause of cervical cancer. Globally, HPV infection has been involved in more than $99 \%$ of cervical cancer, including both cervical squamous cell carcinoma and cervical adenocarcinoma. HPV is a non-enveloped, double-stranded DNA virus, with a genome of approximately $8.0 \mathrm{~kb}$. As of 9 March 2015, more than 200 different types of HPV, identified numerically, have been identified by the International HPV Reference Center[5]. Based on epidemiological and biological data, 
twelve HPV types $(16,18,31,33,35,39,45,51,52,56,58$ and 59$)$ have been classified as HR-HPV by the International Agency for Research on Cancer[6]. Eight HPV types $(26,53,66,67,68,70,73$ and 82$)$, identified as single HPV infections in about $3 \%$ cervical cancer, are classified as probable/possible (p) HR-HPV due to lack of biological data[7]. It is extremely difficult to isolate and culture HPV in vitro. Additionally, not all patients infected with HPV have an obvious antibody response. Thus, HPV DNA detection by PCR becomes a non-invasive and sensitive method for the confirmation of an active cervical HPV infection. Since persistent infection with HR-HPV is a necessary cause for cervical cancer development, DNA detection and genotyping of HPV can be used as an essential method for the control and prevention of HPV-related disease.

Up to present, three licensed prophylactic vaccines, a bivalent vaccine against HPV16 and 18, a quadrivalent vaccine against HPV 6, 11, 16 and 18 and a 9-valent vaccine against HPV 6, 11, 16, 18, 31, 33, 45, 52 and 58, are considered as effective and safe for prevention HPV infection. However, the current vaccines offer protection only targeted at a few HPV genotypes. What's more, virus-type restricted protection is present between HPV genotypes. Since the prevalence of HPV infections in women shows geographical distribution between countries and regions[8, 9], a better understanding of HPV prevalence and genotype distribution would be necessary and valuable for evaluating the effect of the current HPV vaccination program for cervical cancer and developing the next generation of vaccines, particularly in specific regions and areas where they are most needed.

As the data on HPV epidemiology and genotype distribution is lacking in northern Henan Province, which is located in the central China and has the largest population in China, it is impossible to correctly evaluate the prevalence of HPV infection and consequently it is difficulty to take preventive measures, such as the introduction of HPV vaccine in these areas. Therefore, the prevalence and genotypic distribution of HPV among women in Henan Province were identified in this study. The results of this research are of great importance for estimation of the awareness of HPV infection and the introduction of vaccination program in northern Henan Province of China.

\section{Materials And Methods}

\section{Study population and specimen collection}

Women aged between 16 and 81 attending regular gynecological outpatient clinic in Xinxiang central hospital between January 2018 and December 2019 in northern Henan Province were invited to join in this study. They were divided into six aged groups. The G1 group meant age $₫ 20$; the $\mathrm{G} 2$ group meant $20 \leq$ age $₫ 30$; the G3 group meant 30 $\leq$ age $₫ 40$; the $\mathrm{G} 4$ group meant $40 \leq$ age $\$ 50$; the $\mathrm{G} 5$ group meant $50 \leq$ age 660 ; the $\mathrm{G} 6$ group meant age $\geq 60$. Women were excluded for: the presence of cervical cancer, pregnancy at the time of enrollment, previous HPV vaccination, without age information, hysterectomy or immunosuppression. Finally, a total of 15616 participants were included in this study for analysis.

Cervical specimens were collected by the trained clinicians in Xinxiang central hospital. Cervical cells were collected from the cervical canal by using plastic brush. The brush was placed into a $2 \mathrm{~mL}$ vial of preservation solution (Hybribio limited Corp, Chaozhou, Guangdong, China) for HPV DNA detection. This study was performed strictly in accordance with the Declaration of Helsinki and approved by the Ethics Committee in Xinxiang Medical University. Informed consent was obtained from all participants before enrollment.

\section{Dna Extraction, Pcr Amplification And Hpv Genotyping}


HPV DNA was extracted from the cervical cells using a DNA extraction kit according to the manufacturer's manual (Hybribio limited Corp, Chaozhou, Guangdong, China). Briefly, the exfoliated cervical cells were first digested by proteinase $\mathrm{K}$. Then the released DNA was obtained through absorption to magnetic glass particles, washed and purified from these particles with the use of the automated nucleic acid extraction instrument (Hybribio limited Corp, Chaozhou, Guangdong, China). A water blank was performed through all steps of DNA extraction as a contamination control. The concentration and purity (OD260/OD280 between $1.6 \sim 1.8$ ) of DNA were determined by Nanodrop 2000 (ThermoFisher Scientific, CA, USA). The extracted DNA was detected immediately or stored at $-20^{\circ} \mathrm{C}$.

HPV DNA amplification and genotyping were conducted using a commercial HPV genotyping kit for 37 HPV types, including 17 HR-HPV types $(16,18,26,31,33,35,39,45,51,52,53,56,58,59,66,68,82)$ and 20 LR-HPV types $(6,11$, $34,40,42,43,44,54,55,57,61,67,69,70,71,72,73,81,83,84)$. The kits have been authorized by the China Food and Drug Administration for clinical use. The experimental procedures, including PCR and flow-through hybridization, were followed the manufacturer's manual. The HPV L1 consensus biotinylated primer sets were used in the PCR assay. One $\mu \mathrm{L}$ of the DNA was used in the $25-\mu \mathrm{L}$ PCR. PCR reaction was initiated denaturation at $95^{\circ} \mathrm{C}$ for 9 min, followed by 40 cycles of denaturation at $95^{\circ} \mathrm{C}$ for $20 \mathrm{~s}$, annealing at $55^{\circ} \mathrm{C}$ for $30 \mathrm{~s}$ and elongation at $72{ }^{\circ} \mathrm{C}$ for $30 \mathrm{~s}$, with a final extension at $72{ }^{\circ} \mathrm{C}$ for $5 \mathrm{~min}$.

HPV genotyping was performed by flow-through hybridization method. The flow-through hybridization was performed on a medical nucleic acid hybridization instrument prewarmed at $45^{\circ} \mathrm{C}$ prior to usage. A nylon membrane on which 37 HPV genotype-specific oligonucleotide probes secured was placed into the instrument. The biotinylated PCR product was denatured at $95^{\circ} \mathrm{C}$ for $5 \mathrm{~min}$ and then chilled on ice for $2 \mathrm{~min}$ before hybridization. The PCR product was mixed with the hybridization solutions and the mixture was added into the sample wells to conduct with flow-through hybridization for $10 \mathrm{~min}$. The nylon membrane was washed with the hybridization solution three times and the empty region was blocked without reaction. The hybridizing signal were detected with streptavidin alkaline phosphatase, binding to biotinylated PCR products, and its subtract NBT/BCIP (nitro-blue tetrazolium-5-bromo-4-chloro-3indolylphosphate). The genotype result was detected by the position of the HPV-genotype probes on the membrane. The blue dot on the membrane, indicating a positive result, was judged by the naked eyes. Multiple dots showed multiple infections. Quality controls were carried out throughout the whole experiment, including PCR amplification and hybridization by using positive and negative controls provided by the kit.

\section{Statistical analysis}

All statistical analyses were conducted using SPSS 18.0 for Windows (SPSS Inc., Illios, USA). HPV prevalence and genotype distribution were analyzed. Single, double and multiple HPV infections were defined as infection with one, two and more than two genotypes of infections, respectively. HPV prevalence in designated groups and corresponding $95 \%$ confidence intervals $\left(95 \% \mathrm{Cl}\right.$ ) were calculated. The chi square $\left(\chi^{2}\right)$ test was used to evaluate the significance of difference between different age groups. $P<0.05$ was considered to be statistically significant.

\section{Results}

\section{Overall and age-specific HPV prevalence}

From January 2018 to December 2019, a total number of 15616 cervical specimens, collected for HPV DNA detection, were used for statistical analysis. There were 3081 specimens positive for any HPV DNA and the overall prevalence of HPV was 19.7\% (95\% Cl囚19.1\% 20.4\%). The 15616 participants were divided into six age groups and the HPV infection rate in each group was calculated as shown in Table 1. HPV infection lied in each age group, 
however, the infection rates were not the same, and the difference had statistical significance $(P<0.05)$ by using $\chi 2$ test. The highest prevalence of HPV infection was found among women in G1 group with the infection rate of $41.8 \%$ (95\% Cl: $36.5 \%$ 47.2\%), followed by an infection rate of $22.9 \%$ (95\% Cl: $20.2 \% \sim 25.5 \%$ ) in G6 group and an infection rate of $21.1 \%$ (95\% Cl: $20.2 \%$ 25.5\%) in G2 group. HPV prevalence was 18.1\% (95\% Cl: 17.0\% 19.3\%) among women in G3 group, 17.7\% (95\% Cl: 16.6\% 18.8\%) among women in G4 group and 20.8 (95\% Cl: 19.2\% 22.4\%) among women in $\mathrm{G} 5$ group.

\section{Distribution of single, double and multiple HPV infections}

The single, double and multiple HPV infections in different age groups were shown in Table 2. Single, double and multiple HPV infections accounted for 13.7\% (95\% Cl: 13.1\% 14.2\%), 4.3\% (95\% Cl: 4.0\% 4.6\%) and 1.8\% (95\% Cl: $1.6 \% \sim 2.0 \%$ ) of the 15616 cases, respectively. Single genotype HPV infection was the most common pattern, and it occurred more frequently than double and multiple HPV infections. There were statistically significant differences in the age groups distribution of single infections $(P<0.05)$. The group with the highest infection rate was $\mathrm{G} 1 \mathrm{group}$ (28.0\%, 95\% Cl: 36.5\% 47.2\%). The infection rates declined as the age increased, reached the lowest levels in G4 group, and rose again in $\mathrm{G} 6$ group. Statistically significant differences were observed in the age groups distribution of double infections $(P<0.05)$. Starting from $\mathrm{G} 1$ group with the highest infection rate of $10.8 \%$, the infection rate reduced to the lowest levels in G3 group, and then began to increase again from G4 group. Significant differences were also observed in the age groups distribution of multiple infections $(P<0.05)$, with the highest infection rate of $3.1 \%$ in $\mathrm{G} 1$ group and the lowest infection of $1.3 \%$ in $\mathrm{G} 4$ group.

\section{HPV genotype distribution}

There were 36 different HPV genotypes, including 17 HR-HPV genotypes and 19 LR-HPV genotypes, identified in this study. The prevalence of 17 HR-HPV was demonstrated in Table 3. The most common HR-HPV identified was HPV16 (4.3\%), followed by HPV52 (3.5\%), HPV58 (2.0\%), HPV53 (1.8\%) and HPV39 (1.5\%). To be noted, HPV18 was only the seventh most common HR-HPV genotype to be detected. For individuals with single HR-HPV infection, the genotypes that ranked top five were HPV16, HPV52, HPV58, HPV53 and HPV39. For individuals with double HPV infection, the HR-HPV that ranked top five were HPV16, HPV58, HPV52, HPV39 and HPV53. For individuals with multiple HPV infection, the HR-HPV that ranked top five were HPV52, HPV16, HPV58, HPV51 and HPV53. These data suggested that HPV16 infection was predominant in HPV-positive patients. The prevalence of 19 LR-HPV genotypes was demonstrated in Table 4. The most common LR-HPV identified was HPV6, followed by HPV61, HPV81, HPV54 and HPV11. For individuals with single LR-HPV infection, the most commonly detected genotype was HPV61, followed by HPV54, HPV6, HPV81 and HPV11. For double and multiple HPV-infected individuals, HPV6 and HPV61 were the two most common LR-HPV. The overall genotypes that ranked top ten were HPV16, HPV52, HPV58, HPV53, HPV39, HPV51, HPV6, HPV18, HPV61 and HPV81. The top six HPV genotypes were all HR-HPV, demonstrating the most infection were caused by HR-HPV in northern Henan province.

\section{Discussion}

China has a vast territory and a large population of more than 1.4 billion. The prevalence of HPV varied greatly in different provinces of China. There have been some studies reported the prevalence and genotype distribution of HPV in different provinces in China[10, 11, 12, 13]. However, there are few reports on HPV prevalence in Henan Province. The present study provided the prevalence and genotype data of HPV from women attending regular gynecological outpatient clinic from January 2018 to December 2019 in northern Henan province of China. Our study showed that the overall HPV infection rate was $19.7 \%$, which was similar to the results from several surveys of HPV prevalence that 
reported in other provinces of China[14, 15, 16]. The prevalence of HPV in northern Henan province was lower than that reported in Shandong Province (28.4\%) and Fujian Province(38.3\%)[17, 18], but higher than Yunnan Province (12.9\%) and Shanxi Province (8.92\%)[10, 19]. The different HPV prevalence in northern Henan Province was expected to be explained by the different economic conditions, different living habits and customs, cultural diversity, sampling strategy, as well as the HPV detection methods. According to the previous reports, the HPV infection rates ranged from 6.7-44.5\% in China[20]. Although the HPV infection rates in northern Henan Province was not the highest in China, more attention should be paid to the women infected with HPV.

This present study provided age-specific HPV infection rates among women. Our results showed than women under 20 years, with the overall infection rate of $41.8 \%$, had the highest infection rate in the six designed groups. The single HPV infection rate, the double HPV infection rate and the multiple HPV infection rate were also the highest in women under 20 years. There are two possible reasons for this phenomenon: one reason is that compared with other groups, the total number of women under 20 years participating in the survey is relatively small. Healthy women under 20 years undergoing normal medical examinations were rarely required to be tested for HPV. Most of them were outpatients and they come to hospital for some medical problems, leading to the high prevalence of HPV. Another reason is that the young women are sexually active and they don't know how to protect themselves, which makes them sensitive to HPV infection. The above two reasons led to the highest infection rate in G1 group. It was reported that young women infected with HPV were temporary, and the virus would be cleared by the immune system in most of the cases[21, 22]. Therefore, the infection rate of HPV will gradually decline with the age increased. In the present study, the prevalence of HPV declined gradually in the middle-aged groups, and slightly increased in G6 group (22.9\%), which indicated that women over 60 years suffered severely from HPV infection. For women over 60 years, their immune system function gradually decreased, therefore, they become susceptible to HPV infection. If the HPV cannot be eliminated by the immune system and persist for several years, this will represent a risk factor for neoplasia development. HPV infection rates ranged from 17.7-21.1\% among women in groups G2 to G5. Since HPV infection is a major etiological factor for the cervical cancer development, the high prevalence rate of HPV indicated the significant burden of cervical cancer in Henan Province. It is a pity that despite we can detect the HPV infection, there is no medical treatment to eliminate HPV infection. Future treatment that could eliminate HPV infection and prevent progression of cervical cancer is of great interest.

Our present study also provided the age-specific distribution of single, double and multiple HPV infection rates. Except the G1 group, the single, double and multiple HPV infection rates of the rest groups were very close. Up to now, there has not been reached an agreement that whether multiple HPV infections increase the risk of cervical cancer than single HPV infection. Some studies have reported that multiple HPV infections had a higher risk with the occurrence and development of cervical cancer than single HPV infections[23, 24]. However, other studies have found that single HPV infections had a greater risk of developing cervical cancer with respect to multiple infections[25, 26]. In our study, the prevalence of single HPV infections is high, accounted for $13.7 \%$ of the total cases, and HPV16 was the most commonly detected genotype in single HPV infection. HPV16 infection is the one with the highest oncogenic risk[26]. Thus, more attention should be paid to single HPV16 infection. The double HPV infection and multiple HPV infection accounted for $4.3 \%$ and $1.8 \%$ of the total cases, respectively. The investigation of double and multiple HPV is of great importance to study the prevalence of HPV and is also of great significance to develop multivalent HPV vaccine.

It is important to know the HPV genotypes distribution since data concerning the distribution of HPV genotypes is concerning with the vaccine development. Persistent infection with HR-HPV is the primary etiological factor for cervical cancer. There is up to 70\% of cervical cancers caused by HPV16 and HPV18, with the remainder of cervical cancers caused by other HR-HPV genotypes. In the present study, the HPV16, HPV52, HPV58, HPV53 and HPV39 were the top five HR-HPV genotypes. HPV16 ranked first in our study and the infection rate reached up to $4.3 \%$. HPV52 and 
HPV58 ranked second and third respectively. Compared with HPV16, both HPV52 and HPV58 had a lower correlation with cervical cancer. The top three genotypes distribution pattern is consistently with the data from several previous Chinese population-based HPV investigations [17, 27, 28]. It was reported HPV18 ranked second in Chinese women between 1991 and 2016[4]. In our report, HPV18 with an infection rate of 1.1\%, ranked seven in the HR-HPV genotypes in the study, which was consistent with the recent results in several other regions in China[17, 19, 29].

What's more, the study also investigated the distribution of LR-HPV. Our study showed that the top five most common LR-HPV genotypes were HPV6, HPV61, HPV81, HPV54 and HPV11. For the LR-HPV types, cervical cancer association is very rate in the general women population. LR-HPV mainly cause genital warts, common warts, flats warts and many other skin lesions[30], which are usually asymptomatic but are sometimes accompanied by inching, burning or bleeding, leading to psychosocial disturbances. To date, the 9-valent HPV vaccine is used in the prevention of infection with HPV6, HPV11, HPV16, HPV18, HPV31, HPV33, HPV45, HPV52 and HPV58. Our results support the recommendation of the 9-valent HPV vaccine for routine vaccination in northern Henan Province. Meanwhile, vaccine against HPV53, HPV39 and HPV51 should also be developed for the women in this area.

The present investigation provided the recent data on the prevalence and genotype distribution of HPV in northern Henan Province, however, limitations existed. One limitation is that the relationship between the incidence of cervical cancer and the different HPV genotypes infection were not collected in this study. Another limitation is that the viral load in the enrolled individuals were not detected. It has been reported that both HPV genotype and viral load in combination show promise for predicting the development of cervical cancer in HPV positive women[24]. In the future, the role of different HPV genotypes infection and viral load on the risk of cervical cancer will be studied.

\section{Conclusions}

In conclusion, the epidemiology and genotype distribution of HPV were investigated in northern Henan Province. A high prevalence of HPV was confirmed in our study and most infections were caused by HR-HPV, which indicated the a high cervical cancer incidence in the region. HPV16, HPV52 and HPV58 were the dominant HR-HPV which were prevalent in northern Henan Province. The results in our study provide important information for cervical cancer screening and vaccination in women in northern Henan Province of China.

\section{Abbreviations}

HPV: Human papillomavirus; HR-HPV: High-risk HPV; LR-HPV: Low-risk HPV; 95\% Cl: 95\% confidence intervals

\section{Declarations}

\section{Acknowledgements}

We thank the individuals who volunteered to participate in the research. We express our gratitude to those who contributed to the publication of the article. We are grateful to those authors who made available the details of the date from their published articles.

\section{Authors' contributions}

XPW, RLS, MYW and LJZ designed and supervised the research. XPW, YS, XFW and GYW acquisition of data. RLS, MYW and LJZ analyzed and interpreted the data. XPW prepared the manuscript. All authors read and approved the final manuscript. 


\section{Funding}

This study were supported by the Natural Science Foundation of China (No.81802837), the Program for Ph.D. Starting Research Funding from Xinxiang Medical University (No. XYBSKYZZ 201616), the Program for Innovative Research Team (in Science and Technology) in University of Henan Province (No. 20IRTSTHN030), the Natural Science Foundation of Henan Province for Distinguished Young Scholars (No. 212300410013) and the Key Scientific Research Projects of Higher Education Institutions in Henan Province (No. 19A320005).

\section{Availability of data and materials}

The data were collected from Xinxiang central hospital in Xinxiang city. We are grateful for their generous help. The data can be free shared. The materials were purchased from Hybribio Biotechnology Limited Corp. Any addition information may be obtained from the corresponding author on a reasonable request.

\section{Ethics approval and consent to participate}

This study was approved by the Ethics Committee in Xinxiang Medical University. Informed consent was obtained from all participants before enrollment.

\section{Consent for publication}

Not applicable. This manuscript do not contain any individual person's data in any form (including individual details, images or videos.

\section{Competing interests}

We declare that we have no financial and personal relationships with other people or organizations that can inappropriately influence our work. The authors declare that they have no competing interests.

\section{Author details}

${ }^{1}$ Henan Key Laboratory of Immunology and Targeted Drugs, School of Laboratory Medicine, Xinxiang Medical University, Xinxiang 453003, Henan, China. ${ }^{2}$ Department of Clinical Laboratory, Xinxiang Central Hospital, Xinxiang 453000, Henan, China.

\section{References}

1. Small W Jr, Bacon MA, Bajaj A, Chuang LT, Fisher BJ, Harkenrider MM, Jhingran A, Kitchener HC, Mileshkin LR, Viswanathan AN, Gaffney DK. Cervical cancer: a global health crisis. Cancer. 2017;123(13):2404-12.

2. Arbyn M, Weiderpass E, Bruni L, de Sanjosé, Saraiya M, Ferlay J, Bray F. Estimates of incidence and mortality of cervical cancer in 2018: a worldwide analysis. Lancet Glob Health. 2020;8(2):e191-203.

3. Olusola P, Banerjee HN, Philley JV, Dasgupta S. Human papilloma virus-associated cervical cancer and health disparities. Cells. 2019;8(6):622.

4. Zhou HL, Zhang W, Zhang CJ, Wang SM, Duan YC, Wang JX, Yang H, Wang XY. Prevalence and distribution of human papillomavirus genotypes in Chinese women between 1991 and 2016: a systematic review. J Infect. 2018;76(6):522-8.

5. Kocian BJ, Bzhalava D, Forslund O, Dillner J, Poljak M. Molecular methods for identification and characterization of novel papillomaviruses. Clin Microbiol Infect. 2015;21(9):808-16. 
6. Galati L, Brancaccio RN, Gupta P, Lohmann E, Robitaille A, Mandishora RSD, Cuenin C, Filotico R, Combes JD, Giuliano AR, Donà MG, Tommasino M, Gheit T. Diversity of human papillomavirus in the anal canal of HIVpositive and HIV-negative men. J Infect. 2021;82(1):112-6.

7. Halec G, Alemany L, Lloveras B, Schmitt M, Alejo M, Bosch FX, Tous S, Klaustermeier JE, Guimerà N, Grabe N, Lahrmann B, Gissmann L, Quint W, Bosch FX, de Sanjose S, Pawlita M. Pathogenic role of the eight probably/possibly carcinogenic HPV types $26,53,66,67,68,70,73$ and 82 in cervical cancer. J Pathol. 2014;234(4):441-51.

8. Bao HL, Jin C, Wang S, Song Y, Xu ZY, Yan X, Li LM, Ning Y, Wang HJ. Prevalence of cervicovaginal human papillomavirus infection and genotypes in the pre-vaccine era in China: a nationwide population-based study. $J$ Infect. 2021;84(2):75-83.

9. Bogale AL, Belay NB, Medhin G, Ali JH. Molecular epidemiology of human papillomavirus among HIV infected women in developing countries: systematic review and meta-analysis. Virol J. 2020;17(1):179.

10. Li Z, Liu F, Cheng S, Shi L, Yan Z, Yang J, Shi L, Yao Y, Ma Y. Prevalence of HPV infection among 28457 Chinese women in Yunnan Province, southwest China. Sci Rep. 2016;6:21039.

11. Luo L, He P, Liu Q, Jiang Y, Zhang Y, Li Q, Li Q, Li S, Yang F, Ling H, Dai X, Li Z, Chen H. Prevalence and genotype distribution of HPV infection among 214,715 women from Southern China, 2012-2018: baseline measures prior to mass HPV vaccination. BMC Infect Dis. 2021;21(1):328.

12. Li H, Li P, Huang L, Sun L, Ren H, Li P. Prevalence characteristics of cervical human papillomavirus (HPV) infection in zhoupu district, shanghai city, China. Virol J. 2020;17(1):84.

13. Zhang P, Liu S, Zhong Z, Hou J, Lin L, Weng R, Su L, Lei N, Hou T, Yang H. Prevalence and genotype distribution of human papillomavirus infection among women in northeastern Guangdong Province of China. BMC Infect Dis. 2018;18(1):204.

14. Ge Y, Zhong S, Ren M, Ge Y, Mao Y, Cao P. Prevalence of human papillomavirus infection of 65,613 women in East China. BMC Public Health. 2019;19(1):178.

15. Xu HH, Lin A, Chen YH, Dong SS, Shi WW, Yu JZ, Yan WH. Prevalence characteristics of cervical human papillomavirus (HPV) genotypes in Taizhou area, China: a cross-sectional study of 37967 women from the general population. BMJ Open. 2017;7(6):e014135.

16. Mai Q, Yang X, Cheng H, Wu G, Wu Z. Prevalence and genotype distribution of human papillomavirus among women with cervical lesions in Shenzhen city, China. Hum Vaccin Immunother. 2021;17(4):965-71.

17. Jiang L, Tian X, Peng D, Zhang L, Xie F, Bi C, Wang R, Wang J, Qi D. HPV prevalence and genotype distribution among women in Shandong Province, China: analysis of 94489 HPV genotyping results from Shandong's largest independent pathology laboratory. PLoS One. 2019;14(1):e0210311.

18. Wu C, Zhu X, Kang Y, Cao Y, Lu P, Zhou W, Zhou H, Zhang Y, Song Y. Epidemiology of human papilloma virus infection among women in Fujian China. BMC Public Health. 2017;18(1):95.

19. Yang J, Wang W, Wang Z, Wang Z, Wang Y, Wang J, Zhao W, Li D, Liu H, Hao M. Prevalence, genotype distribution and risk factors of cervical HPV infection in Yangqu, China: a population-based survey of 10086 women. Hum Vaccin Immunother. 2020;16(7):1645-52.

20. Li K, Li Q, Song L, Wang D, Yin R. The distribution and prevalence of human papillomavirus in women in mainland China. Cancer. 2019;125(7):1030-7.

21. Garbuglia AR, Lapa D, Sias C, Capobianchi MR, Del Porto P. The use of both therapeutic and prophylactic vaccines in the therapy of papillomavirus disease. Front Immunol. 2020;11:188. 
22. Ferrall L, Lin KY, Roden RBS, Hung CF, Wu TC. Cervical cancer immunotherapy: facts and hopes. Clin Cancer Res. 2021; Online ahead of print.

23. Li Y, Wang H, Zhang Y, Jing X, Wu N, Hou Y, Hao C. Correlation between multi-type human papillomavirus infections and viral loads and the cervical pathological grade. Int J Gynaecol Obstet. 2021;152(1):96-102.

24. Adcock R, Cuzick J, Hunt WC, McDonald RM, Wheeler CM. Role of HPV genotype, multiple infections, and viral load on the risk of high grade cervical neoplasia. Cancer Epidemiol Biomarkers Prev. 2019;28(11):1816-24.

25. Li M, Du X, Lu M, Zhang W, Sun Z, Li L, Ye M, Fan W, Jiang S, Liu A, Wang M, Meng Y, Li Y. Prevalence characteristics of single and multiple HPV infections in women with cervical cancer and precancerous lesions in Beijing, China. J Med Virol. 2019;91(3):473-81.

26. Bruno MT, Scalia G, Cassaro N, Boemi S. Multiple HPV16 infection with two strains: a possible marker of neoplastic progression. BMC Cancer. 2020;20(1):444.

27. Zhang J, Cheng K, Wang Z. Prevalence and distribution of human papillomavirus genotypes in cervical intraepithelial neoplasia in China: a meta-analysis. Arch Gynecol Obstet. 2020;302(6):1329-37.

28. Yuan XW, Li YJ, Qiu Q, Luo Z, Zhao X. Prevalence and genotype distribution of human papillomavirus among 9945 women from the Nanhai area of Foshan. BMC Infect Dis. 2019;19(1):71.

29. Zhang C, Cheng Wj, Liu Q, Guang Q, Zhang Q. Distribution of human papillomavirus infection: a population-based study of cervical samples from Jiangsu Province. Virol J. 2019;16(1):67.

30. Egawa N, Doorbar J. The low-risk papillomaviruses. Virus Res. 2017;231:119-27.

\section{Tables}

Table 1 The prevalence of HPV among women in different groups

\begin{tabular}{|c|c|c|c|c|c|}
\hline Group & Age, y & Sample size & Positive No. & Prevalence, \% (95\% Cl) & P Value \\
\hline G1 & $<20$ & 325 & 136 & $41.8 \rrbracket 36.5-47.2 \rrbracket$ & \\
\hline $\mathrm{G} 2$ & $20-29$ & 2874 & 605 & 21.1ه19.6-22.5》 & \\
\hline G3 & $30-39$ & 4222 & 765 & 18.1凶17.0-19.3凶 & \\
\hline G4 & $40-49$ & 4829 & 856 & 17.7ه16.6-18.8》 & $<0.05$ \\
\hline G5 & $50-59$ & 2417 & 502 & 20.8ه19.2-22.4ه & \\
\hline G6 & $>60$ & 949 & 217 & $22.9 \rrbracket 20.2-25.5 \rrbracket$ & \\
\hline Total & & 15616 & 3081 & 19.7ه19.1-20.4区 & \\
\hline
\end{tabular}

Table 2 Single, double and multiple HPV infections in different groups 


\begin{tabular}{|c|c|c|c|c|c|c|c|}
\hline \multirow[t]{3}{*}{ Group } & \multirow[t]{3}{*}{ Sample size } & \multicolumn{2}{|c|}{ Single infection } & \multicolumn{2}{|c|}{ Double infection } & \multicolumn{2}{|c|}{ Multiple infection } \\
\hline & & Positive & Prevalence, \% & Positive & Prevalence, \% & Positive & Prevalence, \% \\
\hline & & No. & $(95 \% \mathrm{Cl})$ & No. & $(95 \% \mathrm{Cl})$ & No. & $(95 \% \mathrm{Cl})$ \\
\hline G1 & 325 & 91 & $28.0 \rrbracket 23.1-32.9 \rrbracket$ & 35 & 10.8囚7.4-14.2】 & 10 & $3.1 \otimes 1.2-5.0 \otimes$ \\
\hline G2 & 2874 & 396 & $13.8 \otimes 12.5-15.0 \rrbracket$ & 151 & 5.3ه4.4-6.1】 & 58 & $2.0 \otimes 1.5-2.5 \rrbracket$ \\
\hline G3 & 4222 & 562 & 13.3凶12.3-14.3凶 & 142 & 3.4区2.8-3.9凶 & 61 & 1.4ه1.1-1.8》 \\
\hline G4 & 4829 & 611 & $12.7 \rrbracket 11.7-13.6 \rrbracket$ & 181 & $3.7 \rrbracket 3.2-4.3 \rrbracket$ & 64 & $1.3 \otimes 1.0-1.6 \rrbracket$ \\
\hline G5 & 2417 & 331 & 13.7凶12.3-15.1凶 & 115 & $4.8 \rrbracket 3.9-5.6 \rrbracket$ & 56 & 2.3凶1.7-2.9凶 \\
\hline G6 & 949 & 143 & 15.1凶12.8-17.3凶 & 43 & $4.5 \llbracket 3.2-5.9 \rrbracket$ & 31 & 3.3凶2.1-4.4区 \\
\hline Total & 15616 & 2134 & 13.7ه13.1-14.2】 & 667 & $4.3 \rrbracket 4.0-4.6 \rrbracket$ & 280 & $1.8 \otimes 1.6-2.0 \otimes$ \\
\hline P Value & & & $<0.05$ & & $<0.05$ & & $<0.05$ \\
\hline
\end{tabular}

Table 4 Distribution of LR-HPV genotypes in study participants 


\begin{tabular}{|c|c|c|c|c|c|c|c|c|c|}
\hline \multirow{3}{*}{$\begin{array}{l}\text { HPV } \\
\text { type } \\
\text { HPV6 }\end{array}$} & \multicolumn{2}{|c|}{ Single infection } & \multicolumn{3}{|c|}{ Double infection } & \multicolumn{3}{|c|}{ Multiple infection } & Total infection \\
\hline & $\begin{array}{l}\text { Positive } \\
\text { No. }\end{array}$ & $\begin{array}{l}\text { Prevalence, \% } \\
(95 \% \text { Cl) }\end{array}$ & \multicolumn{2}{|c|}{$\begin{array}{l}\text { Positive } \\
\text { No. }\end{array}$} & $\begin{array}{l}\text { Prevalence, } \\
\% \\
(95 \% \mathrm{Cl})\end{array}$ & \multicolumn{2}{|c|}{$\begin{array}{l}\text { Positive } \\
\text { No. }\end{array}$} & $\begin{array}{l}\text { Prevalence, } \\
\% \\
(95 \% \mathrm{Cl})\end{array}$ & \multirow{2}{*}{$\begin{array}{l}\text { Prevalence, } \\
\% \\
(95 \% \mathrm{Cl}) \\
1.4 \rrbracket 1.2- \\
1.6 \rrbracket\end{array}$} \\
\hline & 78 & \multicolumn{2}{|c|}{$0.5 \llbracket 0.4-0.6 \rrbracket$} & 88 & \multicolumn{2}{|c|}{$0.6 \rrbracket 0.4-0.7 \rrbracket$} & 52 & $0.3 \bowtie 0.2-0.4 \rrbracket$ & \\
\hline HPV61 & 111 & \multicolumn{2}{|c|}{$0.7 \rrbracket 0.6-0.8 \rrbracket$} & 27 & \multicolumn{2}{|c|}{$0.2 \rrbracket 0.1-0.2 \rrbracket$} & 30 & $0.2 \varangle 0.1-0.3 \rrbracket$ & $\begin{array}{l}1.1 \otimes 0.9- \\
1.2 \rrbracket\end{array}$ \\
\hline HPV81 & 70 & \multicolumn{2}{|c|}{$0.4 \llbracket 0.3-0.6 \rrbracket$} & 55 & \multicolumn{2}{|c|}{$0.4 \llbracket 0.3-0.4 \rrbracket$} & 43 & $0.3 \rrbracket 0.2-0.4 \rrbracket$ & $\begin{array}{l}1.1 \rrbracket 0.9- \\
1.2 \rrbracket\end{array}$ \\
\hline HPV54 & 79 & \multicolumn{2}{|c|}{$0.5 \rrbracket 0.4-0.6 \rrbracket$} & 51 & \multicolumn{2}{|c|}{$0.3 \rrbracket 0.2-0.4 \rrbracket$} & 32 & $0.2 \varangle 0.1-0.3 \rrbracket$ & $\begin{array}{l}1.0 \otimes 0.9- \\
1.2 \rrbracket\end{array}$ \\
\hline HPV11 & 49 & \multicolumn{2}{|c|}{$0.3 \rrbracket 0.2-0.4 \rrbracket$} & 35 & \multicolumn{2}{|c|}{$0.2 \varangle 0.1-0.3 \rrbracket$} & 32 & $0.2 \varangle 0.1-0.3 \rrbracket$ & $\begin{array}{l}0.7 \rrbracket 0.6- \\
0.9 \rrbracket\end{array}$ \\
\hline HPV40 & 48 & \multicolumn{2}{|c|}{$0.3 \rrbracket 0.2-0.4 \rrbracket$} & 23 & \multicolumn{2}{|c|}{$0.1 \rrbracket 0.1-0.2 \rrbracket$} & 9 & $0.1 \rrbracket 0.0-0.1 \rrbracket$ & $\begin{array}{l}0.5 \rrbracket 0.4- \\
0.6 \rrbracket\end{array}$ \\
\hline HPV84 & 27 & \multicolumn{2}{|c|}{$0.2 \varangle 0.1-0.2 \rrbracket$} & 26 & \multicolumn{2}{|c|}{$0.2 \bowtie 0.1-0.2 \rrbracket$} & 25 & $0.2 \varangle 0.1-0.2 \rrbracket$ & $\begin{array}{l}0.5 \rrbracket 0.4- \\
0.6 \rrbracket\end{array}$ \\
\hline HPV34 & 27 & \multicolumn{2}{|c|}{$0.2 \varangle 0.1-0.2 \rrbracket$} & 14 & \multicolumn{2}{|c|}{$0.1 \otimes 0.0-0.1 \rrbracket$} & 15 & $0.1 \llbracket 0.0-0.1 \rrbracket$ & $\begin{array}{l}0.4 \llbracket 0.3- \\
0.5 \rrbracket\end{array}$ \\
\hline HPV70 & 16 & \multicolumn{2}{|c|}{$0.1 \otimes 0.1-0.2 \rrbracket$} & 16 & \multicolumn{2}{|c|}{$0.1 \rrbracket 0.1-0.2 \rrbracket$} & 10 & $0.1 \rrbracket 0.0-0.1 \rrbracket$ & $\begin{array}{l}0.3 \rrbracket 0.2- \\
0.4 \rrbracket\end{array}$ \\
\hline HPV44 & 20 & \multicolumn{2}{|c|}{$0.1 \varangle 0.1-0.2 \rrbracket$} & 12 & \multicolumn{2}{|c|}{$0.1 \varangle 0.0-0.1 \rrbracket$} & 9 & $0.1 \varangle 0.0-0.1 \rrbracket$ & $\begin{array}{l}0.3 \rrbracket 0.2- \\
0.3 \rrbracket\end{array}$ \\
\hline HPV42 & 21 & \multicolumn{2}{|c|}{$0.1 \rrbracket 0.1-0.2 \rrbracket$} & 9 & \multicolumn{2}{|c|}{ 0.1区0.0-0.1区 } & 8 & $0.1 \rrbracket 0.0-0.1 \rrbracket$ & $\begin{array}{l}0.2 \varangle 0.2- \\
0.3 \rrbracket\end{array}$ \\
\hline HPV55 & 10 & $0.1 \llbracket 0.0-$ & $.1 \otimes$ & 16 & $0.1 \otimes 0$ & $1-0.2 \rrbracket$ & 3 & $0.0 ه 0.0-0.0 \rrbracket$ & $\begin{array}{l}0.2 \varangle 0.1- \\
0.3 \rrbracket\end{array}$ \\
\hline HPV73 & 12 & $0.1 \llbracket 0.0-$ & $.1 \otimes$ & 5 & $0.0 \otimes 0$ & 0-0.1凶 & 11 & $0.1 \otimes 0.0-0.1 \rrbracket$ & $\begin{array}{l}0.2 \rrbracket 0.1- \\
0.2 \rrbracket\end{array}$ \\
\hline HPV43 & 4 & $0.0 \varangle 0.0-$ & $.1 \rrbracket$ & 8 & $0.1 \otimes 0$ & 0-0.1区 & 9 & $0.1 \bowtie 0.0-0.1 \rrbracket$ & $\begin{array}{l}0.1 \rrbracket 0.1- \\
0.2 \rrbracket\end{array}$ \\
\hline HPV69 & 8 & $0.1 \rrbracket 0.0-$ & $.1 \rrbracket$ & 6 & $0.0 \rrbracket 0$ & 0-0.1区 & 4 & $0.0 \rrbracket 0.0-0.1 \rrbracket$ & $\begin{array}{l}0.1 \rrbracket 0.1- \\
0.2 \rrbracket\end{array}$ \\
\hline HPV57 & 4 & $0.0 \varangle 0.0-$ & 0.18 & 9 & $0.1 \bowtie 0$ & 0-0.1区 & 0 & $0.0 ه 0.0-0.0 \rrbracket$ & $\begin{array}{l}0.1 \rrbracket 0.0- \\
0.1 \rrbracket\end{array}$ \\
\hline HPV83 & 5 & $0.0 \rrbracket 0.0-$ & 0.18 & 3 & $0.0 \rrbracket 0$ & 0-0.0区 & 3 & $0.0 \rrbracket 0.0-0.0 \rrbracket$ & $\begin{array}{l}0.1 \otimes 0.0- \\
0.1 \rrbracket\end{array}$ \\
\hline HPV67 & 3 & $0.0 \varangle 0.0-$ & $0.0 \bigotimes$ & 1 & $0.0 \otimes 0$ & $0-0.08$ & 4 & $0.0 \rrbracket 0.0-0.1 \rrbracket$ & $\begin{array}{l}0.1 \rrbracket 0.0- \\
0.1 \rrbracket\end{array}$ \\
\hline HPV71 & 5 & $0.0 \varangle 0.0-$ & $0.1 区$ & 1 & $0.0 \rrbracket 0$ & $0-0.0 \bigotimes$ & 2 & $0.0 \rrbracket 0.0-0.0 \rrbracket$ & $\begin{array}{l}0.1 \otimes 0.0- \\
0.1 \rrbracket\end{array}$ \\
\hline
\end{tabular}


Due to technical limitations, Table 3 is only available as a download in the Supplemental Files section.

\section{Supplementary Files}

This is a list of supplementary files associated with this preprint. Click to download.

- Table3DistributionofHR.docx 